\title{
IoRL Indoor Location Based Data Access, Indoor Location Monitoring \& Guiding and Interaction Applications
}

\author{
Kareem Ali, Akram Alkhatar, Nawar Jawad, John Cosmas, \\ School of Electronic and Computer Engineering \\ Brunel University London, \\ Uxbridge, Middlesex, UB8 3PH, UK \\ Phone: +44 (0)1895 274000 \\ E-mail: \{kareem.ali, john.cosmas\}@brunel.ac.uk
}

\begin{abstract}
The Long-term evolution (LTE) is a standard for high speed mobile communications and 4G LTE communications is all around the world but the future is all about 5G, which is likely to be an evolution from 4G. This is the next generation of mobile communications technology, which in fact could be ten times faster than the existing $4 \mathrm{G}$ connection that will give a superbly speedy and reliable internet access. The internet of radio light (IoRL) project aims to acquire a safer, more secure, customizable and intelligent building network. This paper presents the solution to the existing issues in the LI-FI systems. There are four essential applications that will be achieved by overcoming the issues. They are Location, Monitoring, Guiding and Interaction Applications. The paper discusses the development of each application and its importance.
\end{abstract}

Keywords - Long-term Evolution; Visible Light Communications; Millimeter Wave Communications; Software Defined Networks;

\section{INTRODUCTION}

Application Layer services are constrained by their capabilities of the wireless network on which they operate. Wireless networks in buildings suffer from congestion, interference, security and safety concerns, restricted propagation and poor indoor location accuracy [6]. This means that restricted bandwidth does not allow fast database access and interaction, poor location accuracy prohibits the use of indoor location monitoring, guiding and data access applications. The internet of radio light (IoRL) project aims to develop a safer, more secure, customizable and intelligent building network that reliably delivers increased throughput greater than $10 \mathrm{Gbps}$ from access points and be able to locate a user with an accuracy of less than $10 \mathrm{~cm}$ [6]. Moreover, it will also minimize the interference and power consumption. The outcome and great benefit from the IoRL project is that more appealing broadband communication services for inside buildings can be developed. An increased $10 \mathrm{Gbit} / \mathrm{sec}$ bitrate can be achieved, and more accurate geo location of less than $10 \mathrm{~cm}$ can be obtained [13]. As shown in figure 1, 5G is the fastest, which is $10 \mathrm{Gbps}$ and the lowest bit rate.

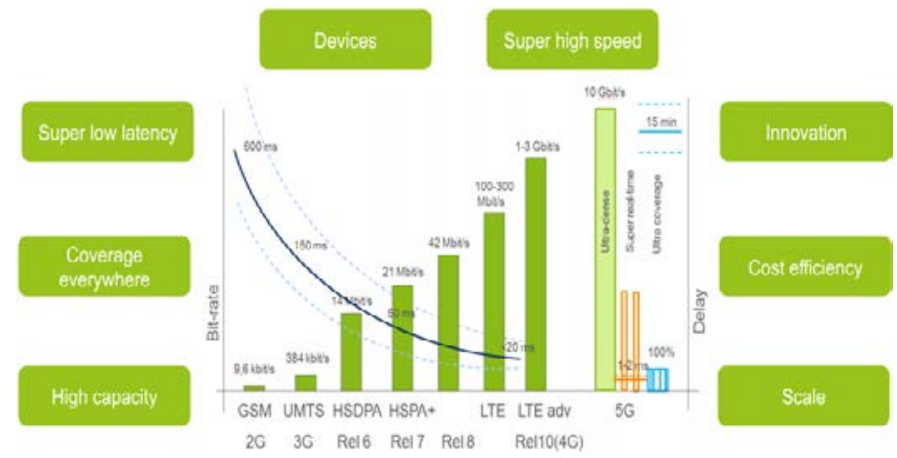

Figure 1: 5G VS other technologies

This paper presents the different applications that can be provided for the home, museum, train station tunnels, supermarket and conference room. At first glance, the service requirements for these widely different indoor environments would seem to be significantly different but on close analysis the new applications that can be developed with these new network capabilities can be grouped into a set of applications that are common to all environments, namely: Indoor Location Based Data Access, Indoor Location Monitoring \& Guiding and Interaction Applications. This paper describes how these common applications for these environments have been developed to have a universal functionality but to have a look-and-feel that is quite different from each other.

\section{ISSUES IN REGARDING EXISTING LI-FI SYSTEMS}

The problem with existing Li-Fi devices are that they are not networked and have location based data stored on USB sticks for each individual light. However, there may be 1000's of Li$\mathrm{Fi}$ access points in for example a museum. Therefore, if the curator wants to update the data on the Li-Fi system, a step ladder is needed to retrieve the USB stick from the Li-Fi access point. In addition, the curator will need to then take the USB card to a PC and update the data manually. Finally, the curator needs to go back and place the USB back into the Li-Fi transmission system. Clearly this is impractical for a museum with 1000's of lights [6]. 


\section{INTERNET OF RADIO LIGHT APPLICATIONS}

What if the above issues discussed previously were resolved? What will it achieve? Presented below, the four crucial applications required.

\section{A. Location Applications}

A location based system would allow for the Museum to deliver data related to an artefact. For a supermarket it would allow data to be delivered related to what is immediately in front of a shopper on the shelves. For a train station it would allow data to be delivered to a traveller on entry to a station or a platform. For a conference it would allow the user to obtain the agenda and proceedings on entry to the conference lobby. For homes it will allow selective control of lights and sensors depending on who is in the room.

\section{B. Monitoring Applications}

Indoor location monitoring would allow the museum to record the behaviour of the visitors. This in turn will benefit the below scenarios.

- The visitor's path through the museum.

- $\quad$ The amount of time each visitor spent at each exhibit.

- $\quad$ The most visited exhibit by visitors.

- The density of visitors in the museum at any one time. In addition, it would allow the services manager in the control office to monitor and locate the maintenance workers that are performing activities in the train station tunnel [12].

\section{Guiding Applications}

Indoor location guiding would allow the visitors with a floor plan to navigate their way around easily. For the train station tunnel, it will allow to guide the maintenance staff to the specific location of the maintenance activity that's taking place. It would allow the shopper at the supermarket to find the relevant route to the products according to their own shopping list.

\section{Interaction Applications}

Interaction would allow the children to play a game "Treasure Hunt" inside the museum, where it provides the children with clues to locate a picture within the museum. The supermarket would provide the customers with an application through a secure IoRL link "Smart Shopping Cart" (SSC), that allows them to prepare the shopping list and find relevant products. In the conference room, the application would allow the delegates to discuss questions and answers, as well as, rating the speakers and the conference.

\section{DESIGN CONCEPT}

There are four main applications (location based download, monitoring, guiding and interacting) that are required for each of the four main scenarios (home, museum, train station and the supermarket). Since these scenarios are quite different they require quite different look and feel but essentially perform the same functionalities. An application will be developed for the user equipment and its interaction with the server applications in the Home IP Gateway (IHIPG) for the various scenarios of the project: Home, Museum, Train Tunnel and Supermarket.
These novel applications for radio-light communication at the Application Layer and Network Layer are unique to RadioLight technology.

- Indoor location based data access (ILBDA), combines four different scenarios, where all can be implemented in a one unique application with different functionalities, as shown in figure 2.

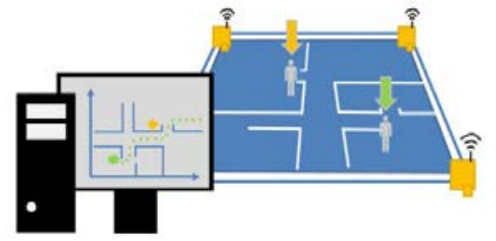

Figure 2: ILBDA

- Indoor location monitoring and guiding (ILMAG), combines the three different scenarios, where all can be implemented by providing a guide and a floor plan assist the users, as shown in figure 3 and figure 4 .

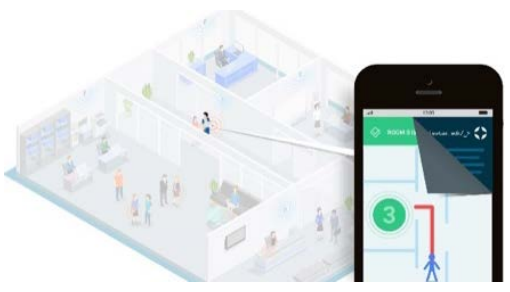

Figure 3 : Indoor Location Monitoring

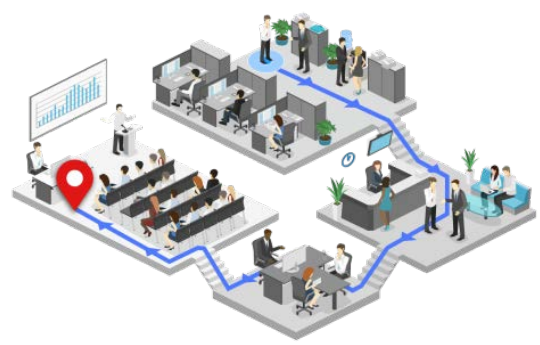

Figure 4 : Indoor Location Guiding

- Interaction includes three different scenarios, where all can be implemented based on the interactions of each of the user, as shown in figure 5.
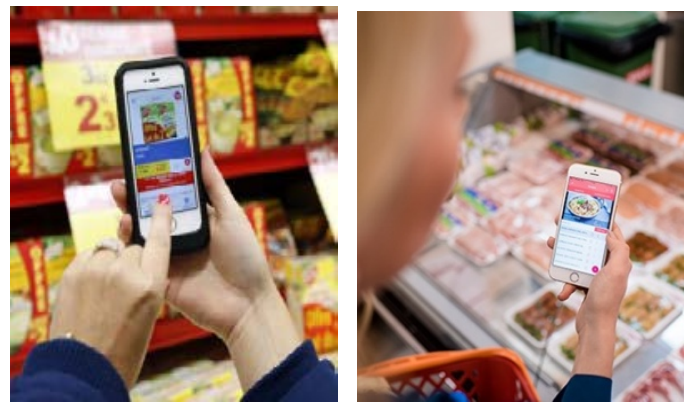

Figure 5 : Interaction 


\section{DEVELOPMENT OF UNIVERSAL APPLICATIONS}

All the following applications require a database that holds the information of the specific scenario. The database has been development for the Museum scenario. However, it can be easily modified to meet the requirements of the other scenarios.

The development of the applications required several different kinds of technologies to be combined. The creation of the application required the use of a database and an interface that will interact with the VLC access points and the database. Essentially the VLC access points would from now on lookup the data from the Database, and the application developed would be able to access and edit the information in this database, making it easier to manage and control the application. The general idea is highlighted with the below diagram, where the Manager edits information in the database, the access points are accessing the database and forwarding the information to the user's device.

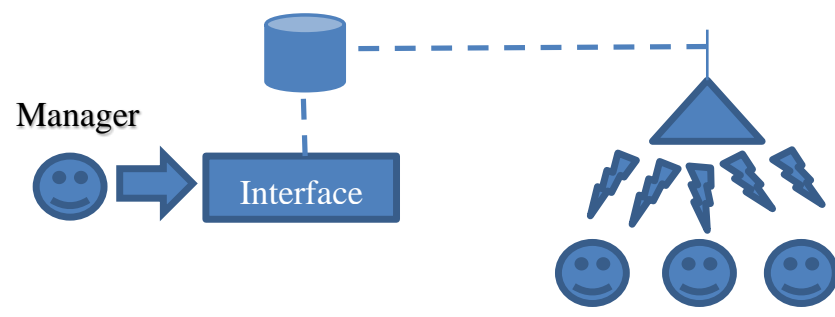

Users

Figure 6: Interaction between manager, database, access points and users

Development of a database driven application such as the above-mentioned program allows the interface (front-end) and database engine (back-end) to work together to provide the VLC access point with the requested data.The front-end and back-end software was developed using Python's Django framework, where the front-end and back-end are used to provide web application with data from the database.

The Django architecture works using modules and the figure 7 below shows the Django architecture on a web server. The different modules within are used to access data from the database and the server provides the information to the frontend.

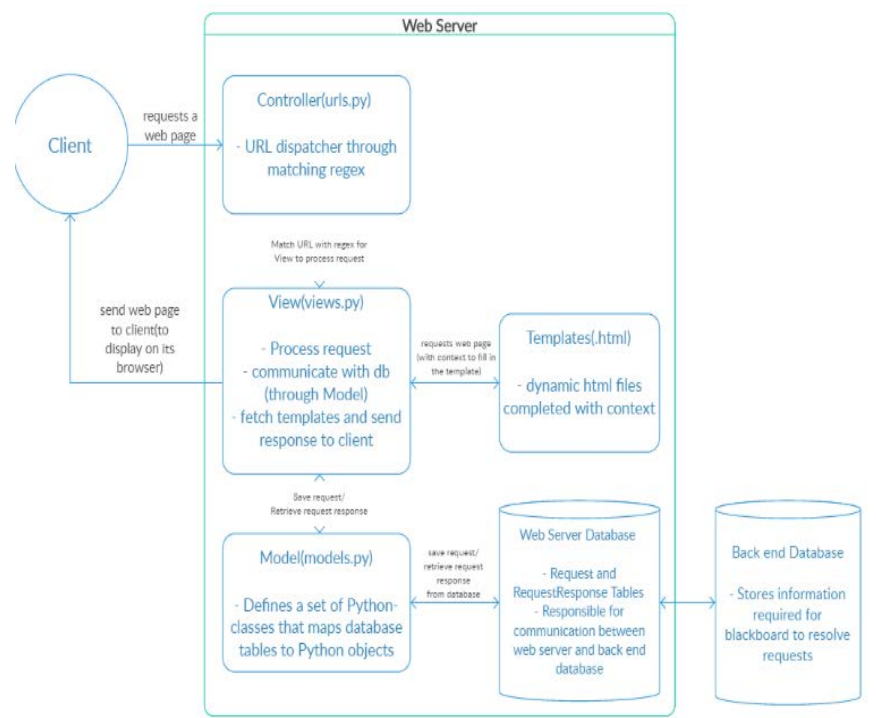

The client initially requests a webpage. The Web Server receives this request and sends the request to the Controller (urls.py module). The Controller then looks at which URL is requested and matches it with the Regular Expressions (REGEX). Once matched with one of the URL patterns, it then requests a view for this URL. This is done at the views.py module. The views module also takes care of implementing any templates that may be required, and any context. If in the Module called 'Model.py' there is information that is required to be loaded, then a connection to the database is made. The views.py module will then put together the template and the information gathered from the database into a response that is then sent back to the client.

The Front-end of the system consists of the following components.

- HTML5 code is served to web browsers, making it universal to all the different platforms whilst staying light and easy to use.

- JavaScript - JavaScript is used to make elements on the web browser interactive without having to reload the webpage.

- CSS3 allows us to make the webpage more appealing, as it has control over how the elements on our pages will look, what color they are and how big.

- Bootstrap framework has been used to provide flexibility in the end system served to both the managers as well as the visitors. It allows the elements to resize depending on the size of the user's screen. This is especially helpful when creating applications for both computers and mobile devices.

- jQuery framework is used to provide interactivity on our web pages. The framework is based on JavaScript.

The back-end primarily consists of the following components:

MySQL - The database engine allows information to be stored by the manager or curator.

Django - Django modules control the whole system and interlink these different platforms.

The front-end and back-end are interlinked using Django. When a request is made, Django uses the various modules to satisfy the request. Part of the request is to get all information and incorporate it all into a front-end response. The front-end being consistent of all these different components is loaded during the response of the request. The response has dynamic content loaded from the database and loaded into the template by one of the modules in Django.

The concept of database driven applications is to have dynamic content that can be easily changed when needed. The different scenarios all require the same functionalities, and the idea of the user interface is to manage the access points and the data that it holds. The different scenarios all require similar functions in their user interfaces. The developed program can be adapted to quickly create a similar program with different needs.

Figure 7 : Django Architecture 


\section{INTERACTION OF APPLICATION LAYER WITH NETWORK LAYER}

This paragraph discusses two of the OSI layers and their interaction together to show the communication between the application and SDN network. The server (database) of the application communicates with the SDN controller through the OSI layers. This process starts at the application layer where it handles the network services; redirector services and name resolution. Redirector services (known as a requester) is a service that looks at the requests that the user may make and check if it can be fulfilled locally if not it will redirect and forward it onto another machine. Name resolution is the process of mapping IP address to a readable name [7]. The network layer is responsible of determining what path a packet would need to take to reach the final destination [9]. The interaction between both layers is conducted by the transport layer as it handles a lot of the interfacing as shown below in figure 8 .

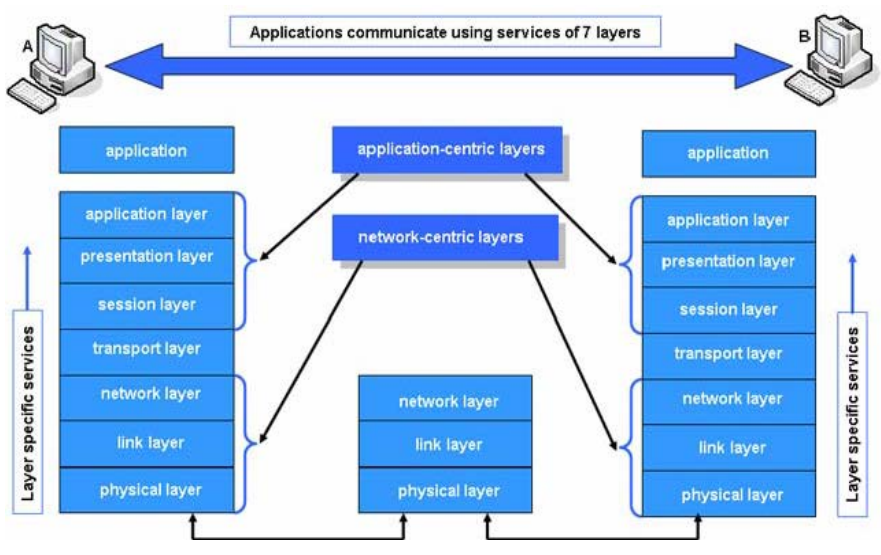

Figure 8 : OSI Layers Model

The web browser loads a lot of different pages of the application and as it is doing so it enquiries the browser about the view size of the device being used. Then the view size is used in the CSS to apply different sizes to the elements in the templates of the system. This same exact method is used to check the browser agent that will return the browser name and type. Moreover, the type of content being displayed is obtained by checking the 'content_type' field in the database for the content being viewed. For example, for videos the value of that field would be 'video_content', which would indicate that a video being streamed.

\section{Create new content}

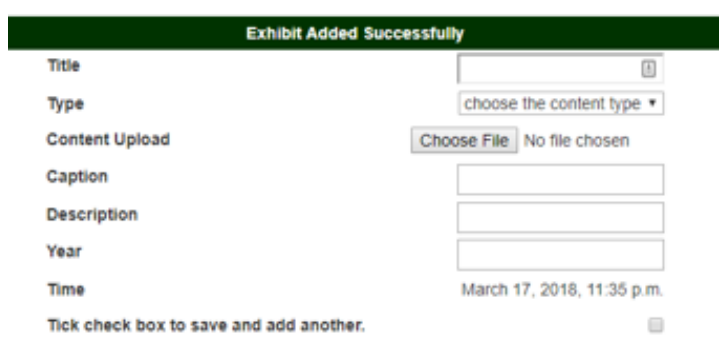

Add Content

Figure 9: Example of the content type on the database
Assuming both WIFI and mobile network are enabled on a device entering a house with the RRLH (Remote Radio Light Head Network). The system is able to detect, which one of them the user is actually using. This would happen by requesting a detailed state of the WIFI information of the device.

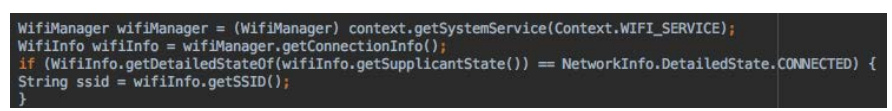

Figure 10 : Finding the state of the WIFI

Figure 11 presents, the IoRL networking layer architecture overview, there will be UEs communicating to the Intelligent Home IP Gateway (IHIPG) using two types of modules, mmWave module and VLC module for up-link-down-link, and down-link respectively. These modules are comprised in the RRLH controllers and traverse the data flows back to IHIPG after going through 5GL1, L2 processing [6]. At the IHIPG, the SDN network implementation is built on Openstack platform and has the Open Daylight (ODL) as the SDN controller, which controls the Open Virtual Stack (OVS) to forward the traffic to the required destination based on the flow matches on IPV4 headers or Ethernet headers. This flow will be destined to the Internet or to the local application servers or back to the MNO, depending on the flow policy requirements.

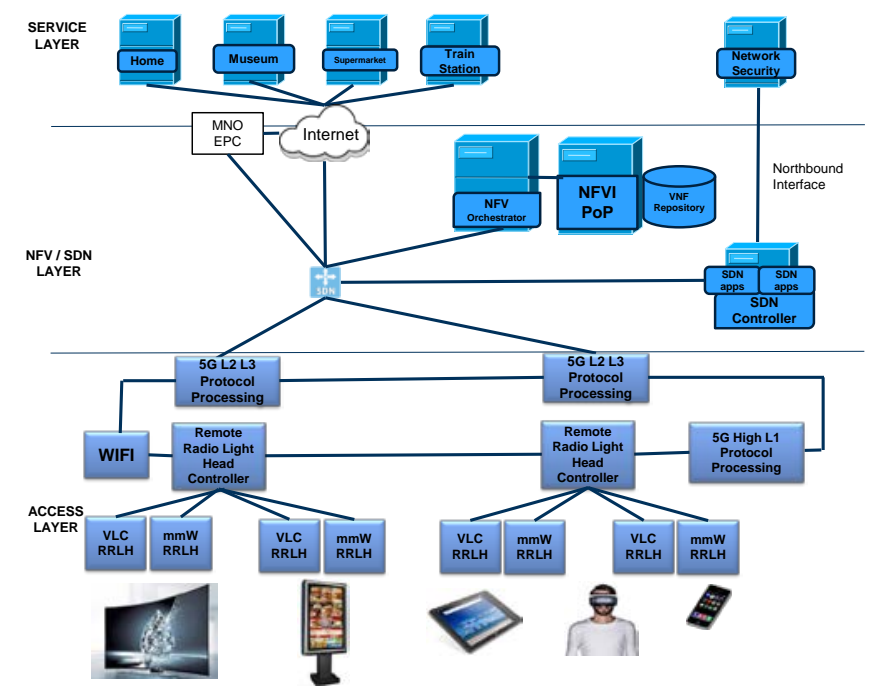

Figure 11: IoRL Networking Layer Architecture

The benefits to 5G Mobile Network Operators (MNOs) by providing by the Network Function Virtualisation (NFV) solutions, is that it provides them with an Application programmers interface (API) [8]. This API allows MNO's to write specialized network applications to manage multi-MNO networks in many places like homes, public space buildings, businesses and environments such as trains and airports. A challenging and critical part of this research will be the abstraction and interface of the mmWave and VLC transceivers within not only the physical design of the 
electronics within existing user products but also within the software and consumer products devices (Application Layer).

The application is being created and developed to not only meet the scenarios requirements but also to be user friendly. This will be a multi-function application, that simplifies all the different scenarios into three main applications.

\section{OTHER APPROACHES}

There are many other technologies that can be used instead of VLC and mmWave like Wi-Fi, BLE, UWB and RFID. However, the available technologies have disadvantages (range, accuracy and indoor tracking) that make them nonfeasible for use indoors. Additionally, there are many applications in the market that were developed to achieve the same outcome, yet, none of them grouped the functionalities of similar scenarios to make it easier for users to use and a lot of them do not have the level of indoor location accuracy to make them feasible.

\section{CONCLUSION}

Location based services are difficult to provide indoors because of the limitations of indoor location-based data access (ILBDA) systems. This paper presents the different applications that can be provided for home, museum, train station tunnels, supermarket and conference room and how these applications could potentially interact with the network layer. This paper described how these common applications for the different environments have been developed to have a universal functionality but to have a look-and-feel that is quite different from each other.

\section{FUTURE WORKS}

People with disabilities are a diverse group in our society. Disability is a multi-dimensional and complex term. As "it denotes impairments, limitations on activity and restrictions on participation" [1]. Some people are born with a disability condition and some others acquire it from a chronic disease or an injury [1]. This project will be able to help people with disabilities worldwide as it develops a communication system through visible light communication. I strongly believe that using VLC technology, would allow to help guide people with disabilities specifically "Vision Disability" while walking using a voice guiding system application as shown in figure 11.

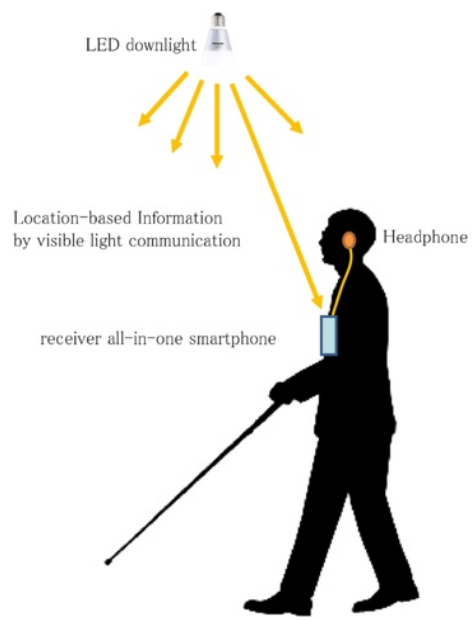

Figure 11: VLC guidance

\section{ACKNOWLEDGEMENTS}

The authors gratefully acknowledge the European Commission for its financial support on the Horizon 2020 Internet of Radio-Light (IoRL) project No: 761992.

\section{REFERENCES}

[1] Europarl.europa.eu. (2015). Assistive technologies to support people with disabilities - Think Tank. [online] Available

at: http://www.europarl.europa.eu/thinktank/en/document.html?re ference=EPRS_BRI(2015)559513 [Accessed 19 Mar. 2018].

[2] H. Elgala, R. Mesleh, and H. Haas, "Indoor optical wireless communication: potential and state-of-the-art," IEEE Communications Magazine, vol. 49, no. 9, pp. 56-62, 2011.

[3] Indoor location monitoring. (2018). [image] Available at: https://www.polestar.eu/wp-

content/uploads/2017/04/sdk@2x.jpg [Accessed 3 Jan. 2018].

[4] Indoor Location. (2018). [image] Available at: https:/cdn.instructables.com/F8E/YIAN/IW6PE10D/F8EYIA NIW6PE10D.MEDIUM.jpg [Accessed 1 Jan. 2018].

[5] Interaction. (2018). [image] Available at: http://static2.businessinsider.com/image/564495c95afbd3fc1d 8b4568-480/a-woman-demonstrates-the-use-of-theapplication.jpg [Accessed 3 Jan. 2018].

[6] J. Cosmas , "Internet of Radio-Light in Buildings Internet of Radio-Light in Buildings,” 01-Jun-2017.

[7] Learning Networking. (2008). How the Application Layer Works. [online] Available at: http://learn-networking.com/tcpip/how-the-application-layer-works [Accessed 12 Apr. 2018].

[8] Qi, W., et al. (2015). "Multiuser MIMO-OFDM for Visible Light Communications." IEEE Photonics Journal 7(6): 1-11.

[9] Reed, D. (2003). Applying the OSI Seven Layer Network Model To Information Security. $1^{\text {st }}$ ed. [pdf] SANS Institute. Available at: https:/luk.sans.org/readingroom/whitepapers/protocols/applying-osi-layer-networkmodel-information-security-1309

[10] T. Komine and M. Nakagawa, "Integrated system of white LED visible light communication and power-line communication” IEEE Trans. Consum. Electron., vol. 49, no. 1, pp. 71-79, Feb. 2003.

[11] vlc. (2018). [image] Available at: http://media.springernature.com/full/springer-

static/image/art\%3A10.1186\%2F1687-1499-2013-

37/MediaObjects/13638_2012_Article_575_Fig1_HTML.jpg [Accessed 1 Jan. 2018].

[11] X. Liu, H. Makino, and Y. Maeda, "Basic study on indoor location estimation using visible light communication platform,” in 2008 30th Annual International Conference of the IEEE Engineering in Medicine and Biology Soci- ety, Aug 2008, pp. 2377-2380.

[12] Zheng, K., et al. (2015). "10 Gb/s hetsnets with millimeter-wave communications: access and networking challenges and protocols." IEEE Communications Magazine 53(1): 222-231. 
